\title{
两亲无规聚合物的聚集行为及其与非离子表面活性剂的相互作用
}

\author{
吴 旭 ${ }^{1,2}$ 乔英杰 $^{1}$ 胡长朝 ${ }^{2}$ 杨 惠 ${ }^{1,2}$ 王金本 ${ }^{2 *}$ \\ (1 哈尔滨工程大学材料科学与化学工程学院, 哈尔滨 150001; \\ 2 中国科学院化学研究所, 胶体、界面与化学热力学院重点实验室, 北京 100190)
}

\begin{abstract}
摘要: 以 2-丙烯酰胺基-十二烷基磺酸 $\left(\mathrm{AMC}_{12} \mathrm{~S}\right)$ 与 2-肉烯酰胺基-2-甲基丙磺酸(AMPS)进行无规共聚, 合成了 含 $\mathrm{AMC}_{12} \mathrm{~S}$ 摩尔分数 $(X)$ 较高 $(X=0.1,0.3,0.5)$ 的一系列两亲聚合物. 采用稳态苂光及动态光散射技术对聚合物在 水溶液中的聚集行为及其与三种非离子表面活性剂 $\left(\mathrm{HO}\left(\mathrm{CH}_{2} \mathrm{CH}_{2} \mathrm{O}\right)_{10} \mathrm{C}_{12} \mathrm{H}_{25}\left(\mathrm{C}_{12} \mathrm{E}_{10}\right) 、 \mathrm{HO}\left(\mathrm{CH}_{2} \mathrm{CH}_{2} \mathrm{O}\right)_{20} \mathrm{C}_{12} \mathrm{H}_{25}\left(\mathrm{C}_{12} \mathrm{E}_{20}\right)\right.$ 和 $\mathrm{HO}\left(\mathrm{CH}_{2} \mathrm{CH}_{2} \mathrm{O}\right)_{40} \mathrm{C}_{12} \mathrm{H}_{25}\left(\mathrm{C}_{12} \mathrm{E}_{40}\right)$ 的相互作用进行了研究, 并考察了 $X$ 对聚集行为的影响以及表面活性剂亲水基 团长度对相互作用的影响. 随着 $X$ 的增大, 聚合物的临界聚集浓度 (CAC)明显减小, $X=0.5$ 时聚合物的 CAC 低 达 $0.0039 \mathrm{~g} \cdot \mathrm{L}^{-1}$. 聚集体的流体力学半径 $\left(R_{\mathrm{h}}\right)$ 都大于 $26 \mathrm{~nm}$, 并随着聚合物浓度的升高而增大, 说明聚合物分子主 要以分子间的聚集方式聚集, 形成多分子聚集体. 随 $X$ 的增大, 聚集体 $R_{\mathrm{h}}$ 减小, 同时 $R_{\mathrm{h}}$ 随聚合物浓度升高而增 大的幅度减小, 说明聚集体结构变得更加紧实. 表面活性剂与聚合物之间存在很强的相互作用, 在混合溶液中表 面活性剂浓度达到临界胶束浓度 $(\mathrm{CMC})$ 左右时聚合物聚集体开始解离, 形成混合聚集体. 亲水基团长度增长, 表 面活性剂对聚合物聚集体的解离能力随之增强. $\mathrm{C}_{12} \mathrm{E}_{40}$ 与 $X=0.5$ 的聚合物形成的混合聚集体 $R_{\mathrm{h}}$ 为 $6.8 \mathrm{~nm}$, 与 $\mathrm{C}_{12} \mathrm{E}_{40}$ 自身形成的聚集体尺寸相当.
\end{abstract}

关键词：非离子表面活性剂；两亲聚合物；聚集行为；聚集体；相互作用 中图分类号: $\mathrm{O} 648$

\section{Aggregation Behavior of Amphiphilic Statistical Polymers and Their Interactions with Nonionic Surfactants}

\author{
WU Xu ${ }^{1,2}$ QIAO Ying-Jie ${ }^{1} \quad$ HU Chang-Chao ${ }^{2} \quad$ YANG Hui $^{1,2} \quad$ WANG Jin-Ben ${ }^{2, *}$ \\ ( ${ }^{1}$ College of Materials Science and Chemical Engineering, Harbin Engineering University, Harbin 150001, P. R. China; \\ ${ }^{2}$ Key Laboratory of Colloid, Interface and Chemical Thermodynamics, Institute of Chemistry, \\ Chinese Academy of Sciences, Beijing 100190, P. R. China)
}

\begin{abstract}
A series of amphiphilic polymers were synthesized by the statistical polymerization of 2-(acrylamido)dodecanesulfonic acid $\left(\mathrm{AMC}_{12} \mathrm{~S}\right)$ with 2-(acrylamido)-2-methylpropanesulfonic acid (AMPS) and they contained a high mole fraction of $\mathrm{AMC}_{12} \mathrm{~S}(X=0.1,0.3,0.5)$. The aggregation behaviors of the polymers and their interactions with three nonionic surfactants $\mathrm{HO}\left(\mathrm{CH}_{2} \mathrm{CH}_{2} \mathrm{O}\right)_{10} \mathrm{C}_{12} \mathrm{H}_{25}\left(\mathrm{C}_{12} \mathrm{E}_{10}\right), \mathrm{HO}\left(\mathrm{CH}_{2} \mathrm{CH}_{2} \mathrm{O}\right)_{20} \mathrm{C}_{12} \mathrm{H}_{25}\left(\mathrm{C}_{12} \mathrm{E}_{20}\right)$, and $\mathrm{HO}\left(\mathrm{CH}_{2} \mathrm{CH}_{2} \mathrm{O}\right)_{40} \mathrm{C}_{12} \mathrm{H}_{25}\left(\mathrm{C}_{12} \mathrm{E}_{40}\right)$ in aqueous solutions were investigated by steady-state fluorescence and quasi-elastic light scattering measurements. The effect of $X$ on the association performance and the influence of the surfactant's hydrophilic group length on these interactions were also investigated. The critical aggregation concentration (CAC) of the polymer was found to significantly decrease with increasing $X$. The CAC of the polymer with $X=0.5$ is as low as $0.0039 \mathrm{~g} \cdot \mathrm{L}^{-1}$. The hydrodynamic sizes of the aggregates $\left(R_{\mathrm{h}}\right)$ are larger than $26 \mathrm{~nm}$ and increase with an increase in polymer concentration, indicating that the polymeric molecules exhibit a strong tendency for interpolymer association, leading to the formation of multimolecular aggregates. When $X$ increases, both $R_{\mathrm{h}}$ and its tendency to increase decrease, suggesting that the aggregates become
\end{abstract}

Received: October 12, 2009; Revised: November 17, 2009; Published on Web: December 14, 2009.

*Corresponding author. Email: jbwang @iccas.ac.cn; Tel/Fax: +86-10-62523395.

The project was supported by the Important National Science and Technology Specific Project of China (2008ZX05024-02) and National High-Tech Research and Development Program of China (863) (2006AA09Z305).

国家科技重大专项(2008ZX05024-02)和国家高技术研究发展计划(863)项目(2006AA09Z305)资助

(C) Editorial office of Acta Physico-Chimica Sinica 
more compact. The interaction between surfactant and polymer is very strong and the polymer aggregates begin to collapse when the surfactant concentration reaches the critical micelle concentration (CMC) in the mixed solutions and mixed aggregates form. The destructive ability of the surfactant increases as the length of the hydrophilic group increases. The $R_{\mathrm{h}}$ value of the mixed aggregates formed by $\mathrm{C}_{12} \mathrm{E}_{40}$ and the polymer with $X=0.5$ is $6.8 \mathrm{~nm}$, which is consistent with the $R_{\mathrm{h}}$ value of the $\mathrm{C}_{12} \mathrm{E}_{40}$ aggregates.

Key Words: Nonionic surfactant; Amphiphilic polymer; Aggregation behavior; Aggregate; Interaction

两亲聚合物以其独特的溶液性质, 在矿物分离、 三次采油、水处理、清洁用品、化妆品及食品业有着 良好的应用前景 ${ }^{[1-3]}$. 由于合成方法不同, 两亲聚合 物可分为嵌段和无规聚合物, 其中无规聚合物的分 子结构比较复杂, 对其研究相对困难 ${ }^{[4]}$. 然而, 已经 用于工业生产的两亲聚合物多是由不同物理、化学 性质的单体通过无规共聚得到的, 这就使对两亲无 规聚合物溶液性质的研究更具实际意义. 目前的研 究表明, 所用单体的种类 ${ }^{[-9]} 、$ 聚合物的组成 ${ }^{[8-13]}$ 、聚合 度 ${ }^{[13]}$ 、疏水基团与分子主链之间的连接基团 ${ }^{[14-180}$ 都是 影响两亲无规聚合物性质的重要因素. 聚合物分子 可能形成分子内的聚集、分子间的聚集或者同时存 在两种聚集. 分子内聚集得到的聚集体为单聚体, 溶 液的粘度随浓度的增加没有明显增加; 而分子间聚 集得到多聚物或空间网状结构, 溶液的粘度随浓度 的增加而明显增加. 我们实验室以较低含量的两亲 单体, 合成了一系列结构新颖、具有优良实用性能的 无规聚合物 ${ }^{[19-20]}$.

虽然对两亲聚合物聚集行为的研究已成为相关 领域的热点, 但是关于含有高含量两亲单体聚合物 的研究却未见报道. 本文以 2-丙烯酰胺基-十二烷基 磺酸 $\left(\mathrm{AMC}_{12} \mathrm{~S}\right.$ )(其摩尔分数 $X=0.1,0.3,0.5$ ) 为疏水基 团, 与 2-丙烯酰胺基-2-甲基丙磺酸(AMPS)进行无 规共聚, 得到系列两亲聚合物(见图 1). 并研究了聚 合物在水溶液中的聚集行为, 探讨 $X$ 对临界聚集 浓度 $(\mathrm{CAC}) 、$ 聚集体流体力学半径 $\left(R_{\mathrm{h}}\right)$ 及 $R_{\mathrm{h}}$ 随浓度

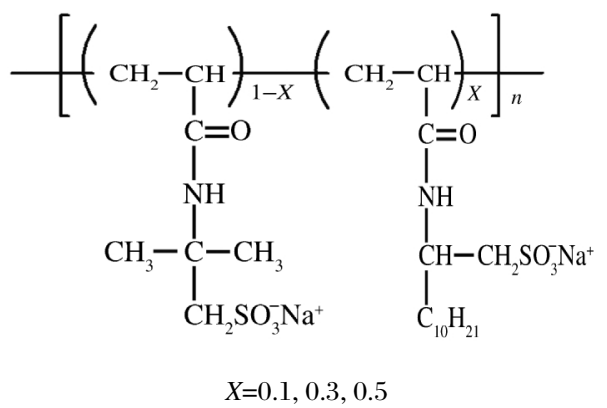

图 1 聚合物的结构式

Fig.1 Chemical structure of the polymers
的变化等性质的影响. 合成三种非离子表面活性剂 $\mathrm{HO}\left(\mathrm{CH}_{2} \mathrm{CH}_{2} \mathrm{O}\right)_{10} \mathrm{C}_{12} \mathrm{H}_{25}\left(\mathrm{C}_{12} \mathrm{E}_{10}\right) 、 \mathrm{HO}\left(\mathrm{CH}_{2} \mathrm{CH}_{2} \mathrm{O}\right)_{20} \mathrm{C}_{12} \mathrm{H}_{25}$ $\left(\mathrm{C}_{12} \mathrm{E}_{20}\right)$ 和 $\mathrm{HO}\left(\mathrm{CH}_{2} \mathrm{CH}_{2} \mathrm{O}\right)_{40} \mathrm{C}_{12} \mathrm{H}_{25}\left(\mathrm{C}_{12} \mathrm{E}_{40}\right)$, 考察聚合物 与表面活性剂之间的相互作用, 并探讨表面活性剂 亲水基团长度对相互作用的影响.

\section{1 实验部分}

\section{1 试剂、合成与表征}

1.1.1 实验试剂

氯化钠、过硫酸铵、硫酸亚铁铵、丙烯腈、发烟硫 酸、月桂醇、氢氧化钾、环氧乙烷、磷酸均为分析纯, 购于北京试剂公司. 十二碳烯纯度高于 $99 \%$, 购于 美国 Fluka 公司. 萠购于美国 Aldrich 公司, 使用前 在乙醇中重结晶. AMPS 纯度高于 $99.4 \%$, 购于山东 寿光市爆源化学有限公司.

\subsection{2 $\mathrm{AMC}_{12} \mathrm{~S}$ 的合成与表征}

$\mathrm{AMC}_{12} \mathrm{~S}$ 合成过程见文献[21]. ${ }^{\mathrm{l}} \mathrm{H}$ 核磁共振 $\left({ }^{1} \mathrm{H}\right.$ NMR)(400 MHz, $\left.\mathrm{D}_{2} \mathrm{O}\right), \delta: 0.83-0.87(\mathrm{t}, 3 \mathrm{H}), 1.25-1.30$ $(\mathrm{m}, 16 \mathrm{H}), 1.53-1.58(\mathrm{~m}, 1 \mathrm{H}), 1.72-1.77(\mathrm{~m}, 1 \mathrm{H}), 3.09$ $(\mathrm{d}, 2 \mathrm{H}), 4.31-4.34(\mathrm{~m}, 1 \mathrm{H}), 5.73-5.76(\mathrm{~d}, 1 \mathrm{H}), 6.15-$ $6.28(\mathrm{~m}, 2 \mathrm{H})$.

\subsection{3 聚合物的合成与表征}

在 $50 \mathrm{~mL}$ 广口瓶中加人 $0.025 \mathrm{~mol}$ 的 $\mathrm{AMC}_{12} \mathrm{~S}$ $(X=0.1 、 0.3 、 0.5)$ 和 AMPS. 加水溶解单体, 控制固体 含量为 $25 \%(\mathrm{w}$, 质量分数). 通人氮气 $10 \mathrm{~min}$, 加人引 发剂 $(7.02 \%(w)$ 硫酸亚铁铵 $2.25 \mathrm{~mL}, 5.88 \%(w)$ 过硫酸 铵 $2.25 \mathrm{~mL}$ ), 体系温度升至大约 $50{ }^{\circ} \mathrm{C}$. 待体系温度 降至室温后, 将产物溶于 $\mathrm{NaOH}$ 的稀溶液中, 并调 节溶液 $\mathrm{pH}$ 值为 7 左右. 用透析袋(北京欣惠泽奥科 技有限公司, 截流相对分子量为 3500)将溶液在水 中透析一周, 冷冻干燥后得到白色固体样品. 元素分 析结果表明产物的碳氮比与投料单体碳氮比相符.

\subsection{4 溶液的配制}

将产物溶解于水中, 并在剧烈搅拌下加热到 90 ${ }^{\circ} \mathrm{C}$. 定量加人 $\mathrm{NaCl}$ 调节溶液离子强度至 $0.05 \mathrm{~mol}$ $\mathrm{L}^{-1}$ 后, 在 $90{ }^{\circ} \mathrm{C}$ 下继续搅拌 $15 \mathrm{~min}$. 在高温剧烈搅拌 
下, 聚合物分子在冷冻干燥过程中形成的相互缠绕 可以完全打开, 并以单分子的形式存在于溶液中. 待 溶液冷却至室温后, 分子重新聚集形成原始的聚集 形态 ${ }^{[22]}$. 样品溶液在进行苂光和动态光散射测试之 前均通过 $0.45 \mu \mathrm{m}$ 薄膜过滤器, 并搅拌 $12 \mathrm{~h}$ 使溶液 达到平衡状态.

\subsection{5 非离子表面活性剂的合成与表征}

向反应釜中加人月桂醇 $18.6 \mathrm{~g}, \mathrm{KOH} 0.213 \mathrm{~g}$, 并密封. 用干燥 $\mathrm{N}_{2}$ 驱替高压釜、进料釜以及进料管 中的空气. 加热升温, 同时用真空泵抽真空, 直到温 度升为 $120{ }^{\circ} \mathrm{C}$. 充 $\mathrm{N}_{2}$ 至釜内压力为正压, 加热至 $150{ }^{\circ} \mathrm{C}$ 时加人环氧乙烷 $44 \mathrm{~g}$. 反应在 $(165 \pm 5){ }^{\circ} \mathrm{C}, 0.3-$ $0.4 \mathrm{MPa}$ 下进行, 直至釜内压力不再降低. 在 $(155 \pm 5)$ ${ }^{\circ} \mathrm{C}$ 下继续反应 $60 \mathrm{~min}$ 后, 用真空泵持续抽真空 30 min. 冷却, 用磷酸中和后得到产物 $\mathrm{C}_{12} \mathrm{E}_{10}$. $\mathrm{C}_{12} \mathrm{E}_{20}$ 和 $\mathrm{C}_{12} \mathrm{E}_{40}$ 的合成路径与 $\mathrm{C}_{12} \mathrm{E}_{10}$ 相同, 反应时分别加人 88 和 $176 \mathrm{~g}$ 的环氧乙烷.

$\mathrm{C}_{12} \mathrm{E}_{10}{ }^{1} \mathrm{H}$ NMR (400 MHz, $\mathrm{CDCl}_{3}$ ), $\delta: 0.83-0.86$ $(\mathrm{t}, 3 \mathrm{H}), 1.23(\mathrm{~s}, 18 \mathrm{H}), 1.51-1.56(\mathrm{~m}, 2 \mathrm{H}), 3.40-3.46(\mathrm{~m}$, $2 \mathrm{H}), 3.52-3.70(\mathrm{~m}, 41 \mathrm{H})$.

$\mathrm{C}_{12} \mathrm{E}_{20}{ }^{1} \mathrm{H}$ NMR (400 MHz, $\mathrm{CDCl}_{3}$ ), $\delta$ : 0.85-0.88 $(\mathrm{t}, 3 \mathrm{H}), 1.24(\mathrm{~s}, 18 \mathrm{H}), 1.53-1.58(\mathrm{~m}, 2 \mathrm{H}), 3.41-3.47(\mathrm{~m}$, $2 \mathrm{H}), 3.55-3.72(\mathrm{~m}, 81 \mathrm{H})$.

$\mathrm{C}_{12} \mathrm{E}_{40}{ }^{1} \mathrm{H}$ NMR (400 MHz, $\mathrm{CDCl}_{3}$ ), $\delta: 0.85-0.87$ (t, 3H), 1.24(s, 18H), 1.52-1.58(m, 2H), 3.41-3.47(m, $2 \mathrm{H}), 3.55-3.72(\mathrm{~m}, 161 \mathrm{H})$.

\section{2 测试方法}

\subsection{1 稳态荧光}

使用配备温度调节装置的 Hitachi F-4500 苂光 仪 (日本 Hitachi 公司) 对样品溶液的苂光强度进行 测定, 测试温度为 $(25.0 \pm 0.5){ }^{\circ} \mathrm{C}$. 激发波长设定为 335 $\mathrm{nm}$, 狭缝宽度为 $2.5 \mathrm{~nm}$. 将 $1 \times 10^{-3} \mathrm{~mol} \cdot \mathrm{L}^{-1}$ 芘的乙醇 溶液注人样品瓶中, 自然挥发掉乙醇溶剂. 将指定浓 度的聚合物溶液注人样品瓶, 菼光测试溶液芘的浓 度保持在 $1 \times 10^{-6} \mathrm{~mol} \cdot \mathrm{L}^{-1}$. 测试前溶液搅拌过夜, 使 溶液达到平衡.

\section{2 .2 动态光散射}

使用配备有 ALV-5000 数字式时间相关器的 ALV/SP-125 激光光散射仪(德国 ALV 公司, 氦-氛 光源, 波长 $632.8 \mathrm{~nm})$ 对样品溶液进行变角度 $\left(30^{\circ}-\right.$ $\left.90^{\circ}\right)$ 的测试, 测试温度为 $(25.0 \pm 0.1){ }^{\circ} \mathrm{C}$. 散射向量 $(q)$ 由公式 $q=(4 \pi n / \lambda) \cdot \sin (\theta / 2)^{[23-24]}$ 得到, 其中 $\theta$ 为散射 角, $n$ 为溶液的折射率. 扩散系数 $(D)$ 通过逆拉普拉
斯变换 $D=\left(\Gamma / q^{2}\right)_{q \rightarrow 0}{ }^{[23-24}$ 计算, 其中 $\Gamma$ 为弛豫率. 流体力 学半径 $\left(R_{\mathrm{h}}\right)$ 由 Stokes-Einstein 公式 $R_{\mathrm{h}}=k_{\mathrm{B}} T /(6 \pi \eta D)^{[23-24]}$ 计算, 其中 $k_{\mathrm{B}}$ 为 Boltzmann 常数, $T$ 为绝对温度, $\eta$ 为溶液粘度.

\section{2 结果与讨论}

苂光光谱可以反映萠探针所处微环境的极性, 从而反映溶液中聚合物形成聚集体的情况 ${ }^{[25]}$. 图 2 为不同浓度聚合物溶液的稳态苂光发射光谱, 随着 聚合物浓度的增加, 谱图中峰 3 的强度 $\left(I_{3}\right)$ 相对于峰 1 的强度 $\left(I_{1}\right)$ 明显增加. 图 3 为不同 $X$ 的聚合物苂光 发射谱图中 $I_{1} / I_{3}$ 对浓度的曲线. 聚合物浓度极稀时 $I_{1} / I_{3}$ 值都在 1.8 左右, 数值与分子萠在水中的 $I_{1} / I_{3}$ 值 (1.8)相符,说明该阶段聚合物以单分子形式存在于 溶液中. 随着聚合物浓度增大, $I_{1} / I_{3}$ 值都在临界聚集 浓度 $(\mathrm{CAC})$ 时迅速减小, 说明分子芘所处微环境的

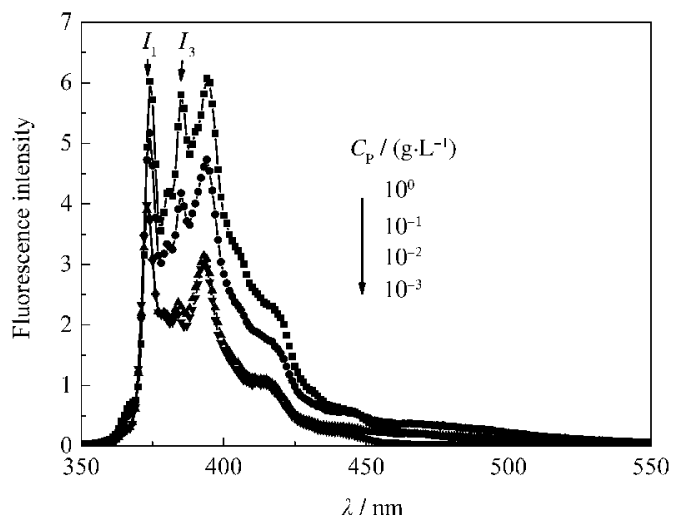

图 $2 X=0.5$ 的聚合物在不同浓度 $\left(C_{\mathrm{P}}\right)$ 下萠的稳态荧光 发射谱图

Fig.2 Steady-state fluorescence emission spectra for pyrene in the presence of varying polymer concentrations $\left(C_{\mathrm{P}}\right)$ with $X=0.5$

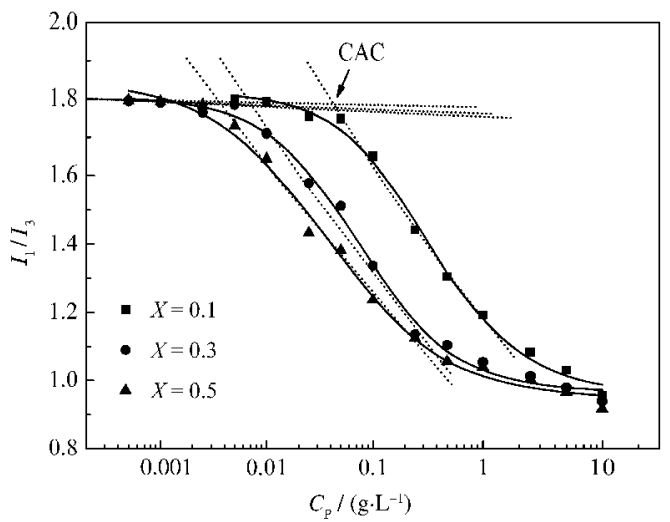

图 $3 I_{1} / I_{3}$ 与聚合物浓度 $\left(C_{\mathrm{P}}\right)$ 之间的关系

Fig.3 Plots of $I_{1} / I_{3}$ ratios as a function of polymer concentration $\left(C_{\mathrm{P}}\right)$ 
表 1 聚合物的临界聚集浓度(CAC)以及在不同聚合物浓 度 $\left(C_{\mathrm{P}}\right)$ 下聚集体的流体力学半径 $\left(\boldsymbol{R}_{\mathrm{h}}\right)$

Table 1 Critical aggregation concentration (CAC) of the polymers and the hydrodynamic sizes of the aggregates $\left(\boldsymbol{R}_{\mathrm{h}}\right)$ at various polymer concentrations $\left(C_{\mathrm{P}}\right)$

\begin{tabular}{cccc}
\hline$X$ & $\mathrm{CAC}\left(\mathrm{g} \cdot \mathrm{L}^{-1}\right)$ & $C_{\mathrm{P}} /\left(\mathrm{g} \cdot \mathrm{L}^{-1}\right)$ & $R_{\mathrm{h}} / \mathrm{nm}$ \\
\hline 0.1 & 0.0467 & 0.2 & 34.3 \\
& & 0.5 & 54.2 \\
& & 1.0 & 69.1 \\
0.3 & 0.0074 & 0.2 & 26.9 \\
& & 0.5 & 33.9 \\
& & 1.0 & 43.1 \\
0.5 & 0.0039 & 0.2 & 26.6 \\
& & 0.5 & 26.9 \\
& & 1.0 & 34.3 \\
\hline
\end{tabular}

极性在迅速减小, 即萠开始溶解在聚合物形成的聚 集体中. $X$ 值越大, $I_{1} / I_{3}$ 值减小所对应的浓度越小, 即聚合物 CAC越小(见表 1).

动态光散射测试中驰豫率(驰豫时间的倒数)是 关于散射角的函数, 随着散射角度的增加, 分子内的 运动会影响驰豫率. 文中聚合物驰豫率与散射向量
平方随着散射角的变化都呈线性关系, 说明该体系 符合分散模型 ${ }^{[23-24]}$, 允许我们计算出 $D$, 进而利用 Stokes-Einstein 公式计算出 $R_{\mathrm{h}}$ (见表 1 ).

图 4 为不同 $X$ 的聚合物在不同浓度下弛豫时 间的分布情况, 相应 $R_{\mathrm{h}}$ 见表 $1 . X=0.5$ 的聚合物在浓 度为 $0.2 \mathrm{~g} \cdot \mathrm{L}^{-1}$ 时 $R_{\mathrm{h}}=26.6 \mathrm{~nm}$, 在聚合物浓度为 1.0 $\mathrm{g} \cdot \mathrm{L}^{-1}$ 时 $R_{\mathrm{h}}=34.3 \mathrm{~nm}$, 聚集体的尺寸增加较小. $X=0.3$ 的聚合物浓度从 $0.2 \mathrm{~g} \cdot \mathrm{L}^{-1}$ 增大到 $1.0 \mathrm{~g} \cdot \mathrm{L}^{-1}, R_{\mathrm{h}}$ 从 $26.9 \mathrm{~nm}$ 增大到 $43.1 \mathrm{~nm} . X=0.1$ 的聚集体尺寸增大 最为明显, $R_{\mathrm{h}}$ 从聚合物浓度为 $0.2 \mathrm{~g} \cdot \mathrm{L}^{-1}$ 的 $34.3 \mathrm{~nm}$ 增大到浓度为 $1.0 \mathrm{~g} \cdot \mathrm{L}^{-1}$ 的 $69.1 \mathrm{~nm}$. 聚集体尺寸的 大小以及尺寸随浓度增大而增大的性质说明聚合物 分子以分子间聚集为主要形式, 形成多分子的聚集 体. $X$ 增大, 聚集体尺寸减小, 同时尺寸随聚合物浓 度升高的增大幅度减小. 由此可以推断出聚集体的 紧实性随着 $X$ 的增大得以增强, 原因可能是聚合物 中疏水基团含量增加, 亲水基团含量减小, 使疏水缔 合作用增强而电荷之间的排斥作用相对减弱, 导致 聚集体的内部结构更为紧实.
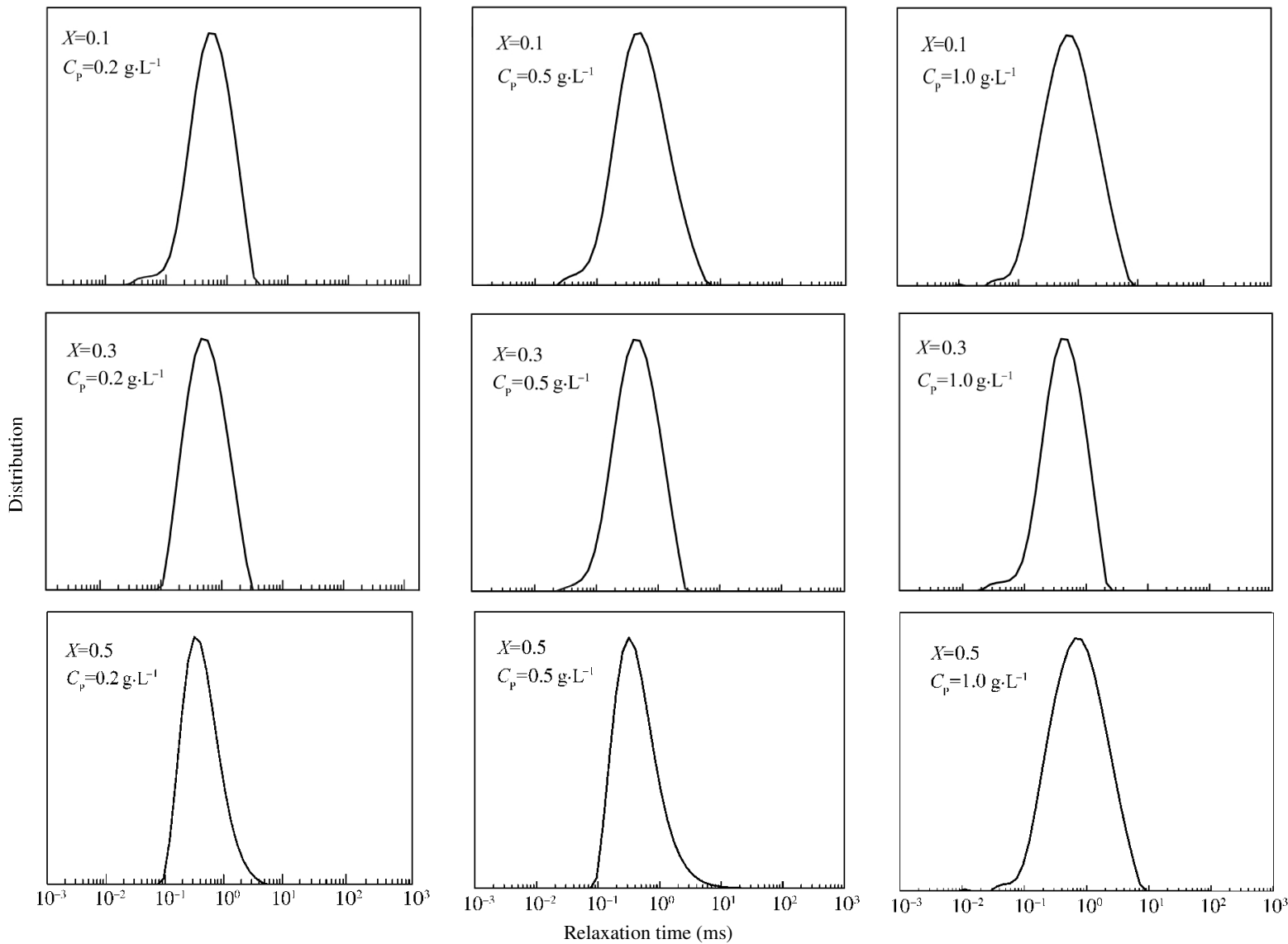

图 4 不同浓度的聚合物在散射角 $\boldsymbol{\theta}=90^{\circ}$ 时的弛豫时间分布

Fig.4 Distributions of relaxation time measured at scattering angle $\theta=90^{\circ}$ for the polymers at different concentrations 


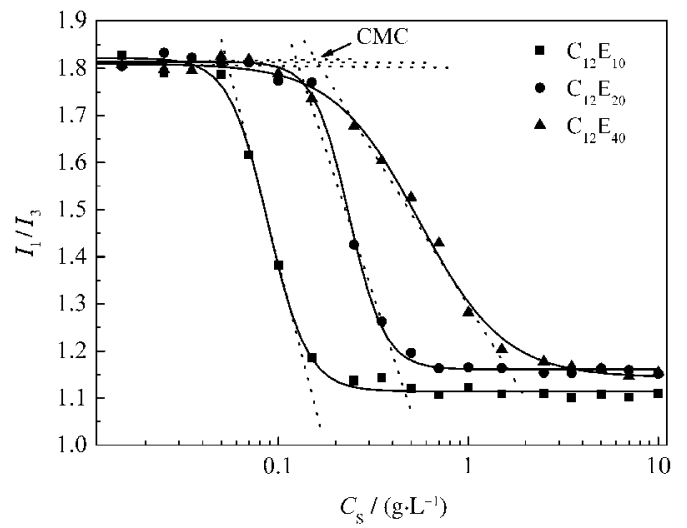

图 $5 I_{1} / I_{3}$ 与表面活性剂浓度 $\left(C_{\mathrm{S}}\right)$ 之间的关系

Fig.5 Plots of $I_{1} / I_{3}$ ratios as a function of surfactant concentration $\left(C_{\mathrm{s}}\right)$

图 5 为三种非离子表面活性剂苂光发射光谱中 $I_{1} / I_{3}$ 对表面活性剂浓度的曲线, 随着非离子表面活 性剂中亲水基团长度的增长, 临界胶束浓度(CMC) 明显增大. 三种表面活性剂 $\mathrm{C}_{12} \mathrm{E}_{10} 、 \mathrm{C}_{12} \mathrm{E}_{20} 、 \mathrm{C}_{12} \mathrm{E}_{40}$ 的 $\mathrm{CMC}$ 分别为 $0.0550 、 0.1279 、 0.1695 \mathrm{~g} \cdot \mathrm{L}^{-1}$.

两亲聚合物与非离子表面活性剂相互作用, 可 使溶液中聚合物聚集体解离, 形成聚合物与表面活 性剂的混合聚集体 ${ }^{[25-28]}$. 图 6 为 $X=0.5$, 浓度为 0.2 $\mathrm{g} \cdot \mathrm{L}^{-1}$ 的聚合物溶液中, $I_{1} / I_{3}$ 值对三种表面活性剂浓 度的曲线, $I_{1} / I_{3}$ 值都呈先增大, 持续某一值后再减小 的变化趋势. 表面活性剂浓度增加到 CMC 左右时, $I_{1} / I_{3}$ 值明显增大, 说明溶液中聚集体极性开始明显

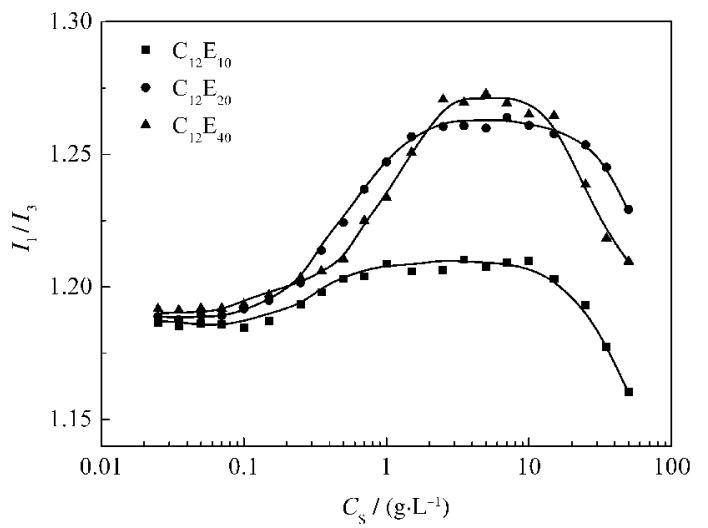

图 $6 I_{1} / I_{3}$ 与聚合物溶液中表面活性剂浓度之间的关系

Fig.6 Plots of $I_{1} / I_{3}$ ratios as a function of the concentration of surfactants in the polymer solution $X=0.5, C_{\mathrm{P}}=0.2 \mathrm{~g} \cdot \mathrm{L}^{-1}$

增大. 聚合物与表面活性剂分子相互作用的增强导 致聚合物分子间作用的减弱, 使部分多分子聚集体 开始解离. 随着表面活性剂的加人, $I_{1} / I_{3}$ 值不变, 此 阶段可能由于溶液中已不存在可解离的多分子聚集 体, 使新增表面活性剂主要以游离状态存在于溶液 中, 浓度的增大对微观极性影响不大. 表面活性剂浓 度继续增大, 新增表面活性剂自身开始聚集形成聚 集体, 使溶液中聚集体数目增多, 整体微观极性减 小, $I_{1} / I_{3}$ 值继续减小.

根据图 6 中 $I_{1} / I_{3}$ 值平稳阶段取表面活性剂浓度 $5.0 \mathrm{~g} \cdot \mathrm{L}^{-1}$, 考察表面活性剂溶液及其与聚合物混合 溶液弛豫时间分布情况如图 7 所示. 从图 7 中混合
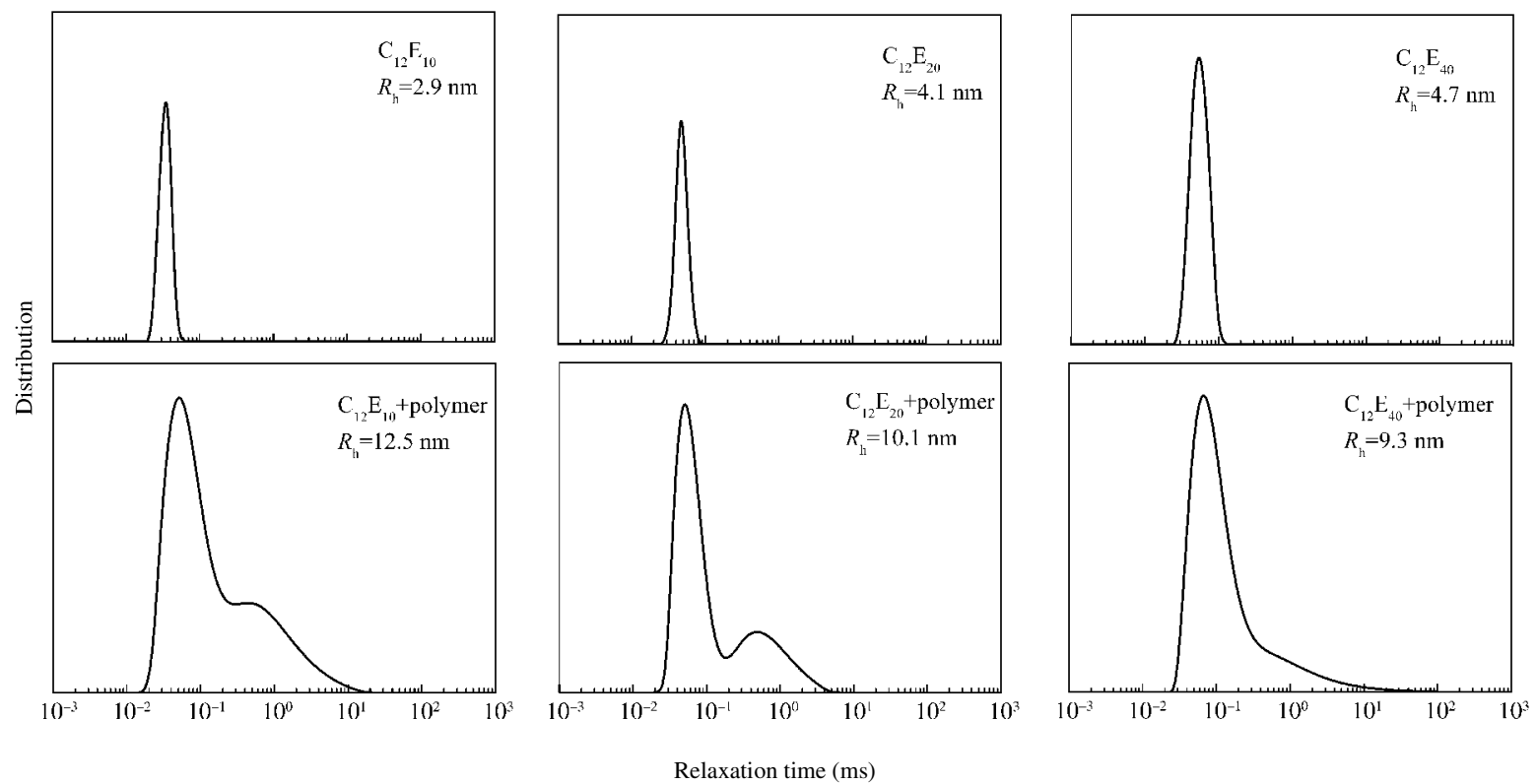

图 7 不同表面活性剂及其与聚合物的混合溶液在散射角 $\theta=90^{\circ}$ 时的弛豫时间分布

Fig.7 Distributions of relaxation time measured at dispersion angle $\theta=90^{\circ}$ for the different surfactant solutions and the mixed solutions of surfactants and polymer $C_{\mathrm{S}}=5.0 \mathrm{~g} \cdot \mathrm{L}^{-1}, X=0.5, C_{\mathrm{P}}=0.2 \mathrm{~g} \cdot \mathrm{L}^{-1}$ 

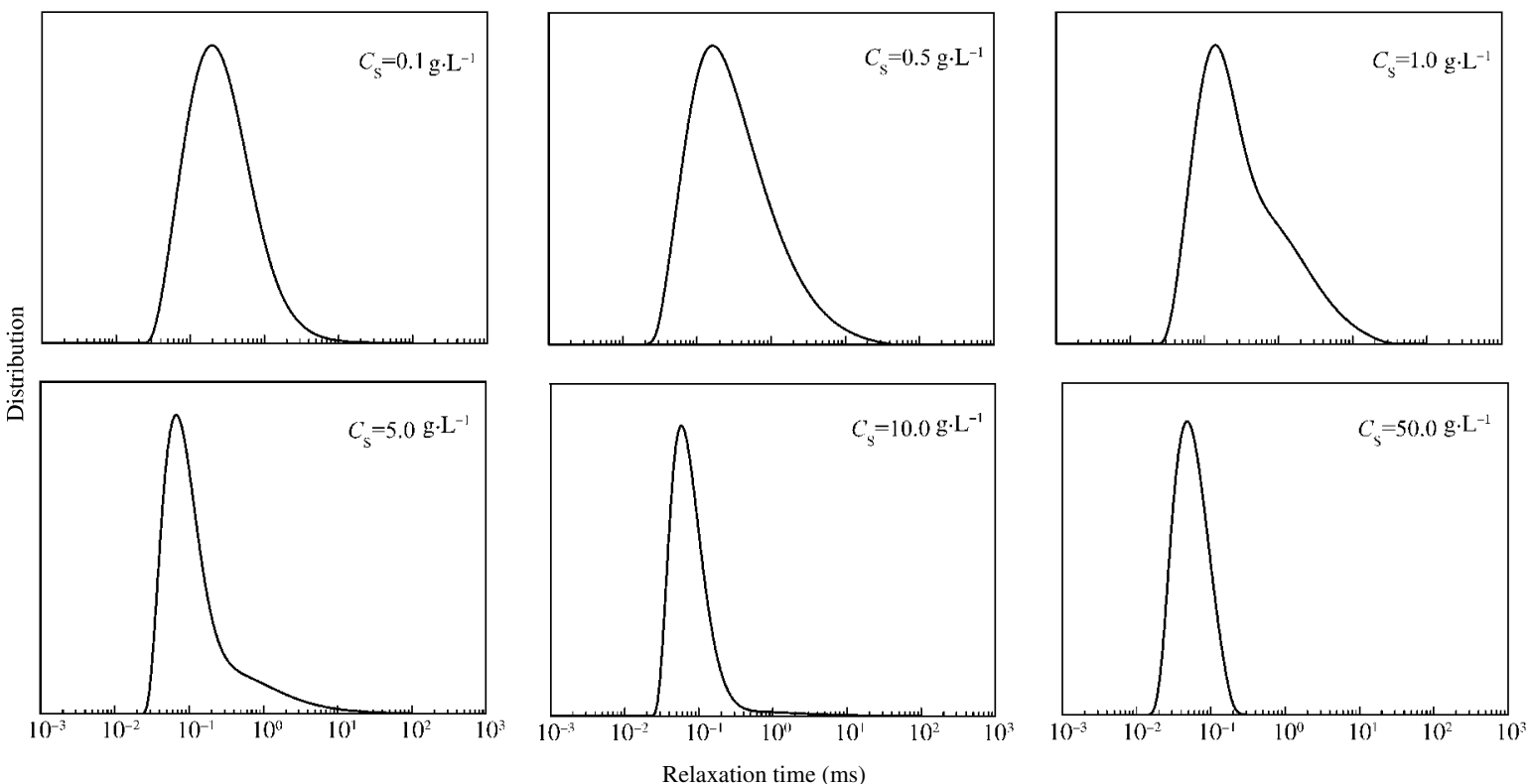

图 8 含有不同 $\mathrm{C}_{12} \mathrm{E}_{40}$ 浓度的聚合物溶液在 $\theta=90^{\circ}$ 时的弛豫时间分布

Fig.8 Distributions of relaxation time measured at $\theta=90^{\circ}$ for the polymer solutions in the presence of $\mathbf{C}_{12} \mathbf{E}_{40}$ at different concentrations $X=0.5, C_{\mathrm{P}}=0.2 \mathrm{~g} \cdot \mathrm{L}^{-1}$

溶液弛豫时间的峰型可以看出, 表面活性剂亲水基 团越长, 混合溶液中大尺寸的聚集体含量越少, 说明 表面活性剂对聚合物聚集体的解离能力随着亲水基 团长度的增长而增大. 原因可能是参与到聚集体中 表面活性剂分子亲水基团越长, 空间位阻越大, 使疏 水缔合的聚合物碳链之间更容易分离. 溶液中仍存 在大尺寸的聚集体, 而此时苂光谱图中 $I_{1} / I_{3}$ 值随表 面活性剂浓度增大变化不大可能是因为少量聚合物 聚集体的解离对体系微观极性影响不大.

图 8 为随 $\mathrm{C}_{12} \mathrm{E}_{40}$ 浓度增加, 混合溶液弛豫时间 分布情况. $\mathrm{C}_{12} \mathrm{E}_{40}$ 浓度为 $1.0 \mathrm{~g} \cdot \mathrm{L}^{-1}$ 时, 弛豫时间的分 布明显变窄, 大尺寸的聚集体开始减少, 说明此时部 分聚合物聚集体开始解离. 当 $\mathrm{C}_{12} \mathrm{E}_{40}$ 浓度增加到 $10.0 \mathrm{~g} \cdot \mathrm{L}^{-1}$, 体系中不存在大尺寸的聚合物聚集体, 此时 $R_{\mathrm{h}}$ 大体反映了聚合物与 $\mathrm{C}_{12} \mathrm{E}_{40}$ 形成混合聚集 体的尺寸, 经计算 $R_{\mathrm{h}}$ 为 $6.8 \mathrm{~nm}$. 当 $\mathrm{C}_{12} \mathrm{E}_{40}$ 浓度增大 到 $50.0 \mathrm{~g} \cdot \mathrm{L}^{-1}$ 时, 相比 $10.0 \mathrm{~g} \cdot \mathrm{L}^{-1}$ 时弛豫时间并无明 显变化, $R_{\mathrm{h}}$ 为 $4.7 \mathrm{~nm}$, 说明混合聚集体与 $\mathrm{C}_{12} \mathrm{E}_{40}$ 自身 形成的聚集体尺寸相当.

\section{3 结 论}

以 $\mathrm{AMC}_{12} \mathrm{~S}$ 与 $\mathrm{AMPS}$ 通过无规共聚合成了系列 两亲聚合物, 其中 $\mathrm{AMC}_{12} \mathrm{~S}$ 摩尔分数 $X=0.1 、 0.3 、 0.5$. 随着 $X$ 的增加, 聚合物 $\mathrm{CAC}$ 明显减小. $X=0.5$ 的聚合
物 CAC 低达 $0.0039 \mathrm{~g} \cdot \mathrm{L}^{-1}$. 聚合物浓度 $0.2 \mathrm{~g} \cdot \mathrm{L}^{-1}$ 时, 聚集体的流体力学半径都大于 $26 \mathrm{~nm}$, 并随浓度的 升高而增大, 说明聚合物分子主要以分子间的聚集 方式聚集, 形成多分子聚集体. 增大 $X$, 使聚合物分 子疏水缔合作用增强而电荷之间的排斥作用相对减 弱, 导致聚集体的内部结构更为紧实. 表现为聚集体 尺寸减小, 同时尺寸随浓度升高的增大幅度减小. 非 离子表面活性剂与聚合物存在很强的相互作用, 在 混合溶液中浓度达到 $\mathrm{CMC}$ 左右时聚合物聚集体开 始解离, 形成混合聚集体. 表面活性剂的亲水基团长 度越长, 空间位阻越大, 使疏水缔合的聚合物碳链之 间更容易分离, 对聚合物聚集体的解离能力越强. $\mathrm{C}_{12} \mathrm{E}_{40}$ 在混合溶液中浓度为 $10.0 \mathrm{~g} \cdot \mathrm{L}^{-1}$ 时将 $0.2 \mathrm{~g} \cdot \mathrm{L}^{-1}$ 、 $X=0.5$ 的聚合物聚集体全部解离, 形成的混合聚集 体的流体力学半径 $R_{\mathrm{h}}$ 为 $6.8 \mathrm{~nm}$, 与 $\mathrm{C}_{12} \mathrm{E}_{40}$ 自身形成 的聚集体尺寸相当.

\section{References}

1 Hurter, P. N.; Scheutjens, J. M. H. M.; Hatton, T. A. Macromolecules, 1993, 26: 5592

2 Glatter, O.; Scherf, G.; Schillen, K.; Brown, W. Macromolecules, 1994, 27: 6046

3 Webber, S. E. J. Phys. Chem. B, 1998, 102: 2618

4 Kawata, T.; Hashidzume, A.; Sato, T. Macromolecules, 2007, 40: 1174 
5 Kathmann, E. E.; White, L. A.; McCormick, C. L. Macromolecules, 1996, 29: 5273

6 Kramer, M. C.; Welch, C. G.; Steger, J. R.; McCormick, C. L. Macromolecules, 1995, 28: 5248

7 Morishima, Y.; Nomura, S.; Ikeda, T.; Seki, M.; Kamachi, M. Macromolecules, 1995, 28: 2874

$8 \mathrm{Hu}$, Y.; Smith, G. L.; Richardson, M. F.; McCormick, C. L. Macromolecules, 1997, 30: 3526

9 Hu, Y.; Armentrout, R. S.; McCormick, C. L. Macromolecules, 1997, 30: 3538

10 Suwa, M.; Hashidzume, A.; Morishima, Y.; Nakato, T.; Tomida, M. Macromolecules, 2000, 33: 7884

11 Branham, K. D.; Snowden, H. S.; McCormick, C. L. Macromolecules, 1996, 29: 254

12 Yamamoto, H.; Mizusaki, M.; Yoda, K.; Morishima, Y. Macromolecules, 1998, 31: 3588

13 Hashidzume, A.; Kawaguchi, A.; Tagawa, A.; Hyoda, K.; Sato, T. Macromolecules, 2006, 39: 1135

14 Yusa, S.; Kamachi, M.; Morishima, Y. Langmuir, 1998, 14: 6059

15 Noda, T.; Morishima, Y. Macromolecules, 1999, 32: 4631

16 Noda, T.; Hashidzume, A.; Morishima, Y. Macromolecules, 2000, 33: 3694

17 Noda, T.; Hashidzume, A.; Morishima, Y. Langmuir, 2000, 16:
5324

18 Noda, T.; Hashidzume, A.; Morishima, Y. Macromolecules, 2001, 34: 1308

19 Xu, X. H.; Yang, F.; Wang, N.; Shen, M. H.; Zhou, T. T.; Wang, X. Y.; Wang, Y. L.; Chen, X. J.; Wang, J. B. Journal of Dispersion Science and Technology, 2008, 29: 101

20 Zhao, Y. Z.; Zhou, J. Z.; Xu, X. H.; Liu, W. B.; Zhang, J. Y.; Fan, M. H.; Wang, J. B. Colloid Polym. Sci., 2009, 287: 237

21 Yu, Y. M.; Gao, B. J.; Wang, R. X. Chin. J. Colloid Polym., 2005, 23: 26 [于亚明, 高保娇, 王芯欣. 胶体与聚合物, 2005, 23: 26]

22 Yamamoto, H.; Tomatsu, I.; Hashidzume, A.; Morishima, Y. Macromolecules, 2000, 33: 7852

23 Wilhelm, M.; Zhao, C. L.; Wang, Y.; Xu, R.; Winnik, M. A.; Mura, J. L.; Riess, G.; Croucher, M. D. Macromolecules, 1991, 24: 1033

24 Hashidzume, A.; Mizusaki, M.; Yoda, K.; Morishima, Y. Langmuir, 1999, 15: 4276

25 Yusa, S.; Hashidzume, A.; Morishima, Y. Langmuir, 1999, 15 8826

26 Yamamoto, H.; Morishima, Y. Macromolecules, 1999, 32: 7469

27 Hashidzume, A.; Ohara, T.; Morishima, Y. Langmuir, 2002, 18: 9211

28 Jacquin, M.; Muller, P.; Cottet, H.; Crooks, R.; Thoédoly, O. Langmuir, 2007, 23: 9939 